\title{
THE DOMINANCE OF DARK ENERGY LEADS TO REDUCTION OF THE ENTROPY OF GALAXIES FLOW AND ENTROPY OF THE UNIVERSE
}

\author{
Aleksandr V. Bukalov \\ The Centre for Physical and Space Research, International Institute of Socionics, \\ Melnikova str., 12, Kyiv-050,04050,Ukraine, bukalov.physics@socionic.info
}

\begin{abstract}
The existence of the Hubble flow recession of galaxies in terms of dominance of dark energy density, vacuum energy, reduces the gravitational entropy of clusters of galaxies, reducing the gravitational entropy of the Universe as a whole. Global dominance of dark energy leads to a decrease in entropy of the Universe within the cosmic event horizon.
\end{abstract}

Keywords: Hubble flow, movement of galaxies, clusters of galaxies, dark energy, entropy of the Universe

The dominance of dark energy in the modern era leads to the dominance of anti-gravitation of gravity on cosmological scales. I.D.Karachentsev (Karachentsev \& Kashlbadze, 2006; Karachentsev, 2005; Karachentsev et al., 2009; Karachentsev, Karachentseva \& Huchtmeier, 2007) and A.D.Chernin (Chernin, Teerikorpi, Baryshev, 2003; Chernin et al., 2007; Chernin et al., 2009; Chernin et al., 2012; Chernin et al., 2013; Chernin et al., 2007; Chernin et al., 2012; Chernin, 2013; Teerikorpi \& Chernin, 2010), studying the motions of galaxies in clusters, have shown that anti-gravity effects manifest themselves not only on a cosmic scale, but the scale of clusters of galaxies, for example - the Local Group, including our galaxy (the Milky Way) galaxy Andromeda and dozens of other smaller galaxies. M. Eingorn and A. Zhuk also studied in detail these issues (Brilenkov, Eingorn \& Zhuk, 2015; Eingorn \& Zhuk, 2012). Observations and modeling have shown that at distances of 1-3 Mpc from the center of the gravity bounded Local Group of galaxies it is observed the local flow of divergent dwarf galaxies. The velocities of these galaxies are proportional to the distance from the center. A.D.Chernin et al. (2012) have shown that such flows, which are observed in other clusters at different scales, form under the influence of dark energy. So, for Local Groups zone of zero gravity in which the gravitational force is balanced by the force of attraction and anti-gravitation is the $R_{Z G} \approx 1.3 \div 1.4 \mathrm{Mpc}$. Therefore, when $R>R_{Z G}$ the flow of recession of galaxies starts. A.D.Chernin and colleagues studied the behavior of galaxies streams, build charts based on experimental data and numerical simulation and showed that the phase trajectories of the local stream tend to phase attractor $V=H_{x} R$, that corresponds to the Hubble law. In this case $H_{x}=\left(8 \pi G \rho_{x} / 3\right)^{1 / 2}$ is determined by the local density of dark energy (Chernin, 2013). Therefore, when $R>R_{Z G}$ flow of receding galaxies starts. In this case the phase trajectories of the local flow tend to phase attractor $V=H_{x} R$, that is, obey the Hubble law. From experimental diagram of velocities of galaxies we can estimate that the spread of own velocities of galaxies with $R<R_{Z G}$ reaches $\Delta V_{\max }= \pm 150 \mathrm{~km} / \mathrm{s}$, the average is $\Delta V= \pm 70 \mathrm{~km} / \mathrm{s}$, while when $R>R_{Z G}$ velocity spread to the theoretical velocity of Hubble divergence flow averages about $\Delta V=12 \mathrm{~km} / \mathrm{s}$ (Bukalov, 2014). Antigravity of dark energy, or the energy of the vacuum, reduces the dispersion of velocity galaxies. The difference in entropy in areas with $R<R_{Z G}$ and $R>R_{Z G}$ is negative, so "evaporation" of the galaxies from gravitationally bound clusters reduces its gravitational entropy. Thus the antigravitation area causes a decrease in entropy of "gas of galaxies" at different levels of the hierarchy, in clusters and superclusters. In fact, gravitationally bound system, such as a group or cluster of galaxies, has gravitational entropy more than a galaxy outside the gravitational cluster and moving in an anti-gravitational environment. The "evaporation" of the galaxies gravitationally bound cluster reduces its gravitational entropy. Thus, the area of anti-gravitation causes a decrease in the entropy "gas galaxies" at different levels of the hierarchy - in clusters and superclusters.

Based on the results of numerical modeling of flow of galaxies recession, in which were obtained the minimum and maximum velocity of flow $V_{\min }=$ $=H_{x} R\left(1+2 x^{-3}-3 x^{-2}\right)^{1 / 2}$ and $V_{\max }=H_{x} R\left(1+3 x^{-1 / 2}\right)$, where $x=R / R_{Z G}$ (Chernin, 2013), we can write in general form the expression for the entropies difference:

$$
\Delta S=\ln \frac{\left(V_{2_{\max }}-V_{2_{\text {min }}}\right)^{2}}{\left(V_{1_{\max }}-V_{1_{\min }}\right)^{2}} .
$$

We have obtained the entropy flow of galaxies similar to the gas. But if we use obtained by Chernin A.D. (2013) vacuum cooling factor as ratio of velocities, the logarithm of the square of this factor also provides an estimate of entropy changes. However, we can consider the more general expression for the evaluation of gravitational entropy of the system. We express the acceleration as equivalent to the Unruh vacuum temperature of accelerated moving body, for example a galaxy:

$$
\frac{\hbar \ddot{a}}{2 \pi c k_{B}}=\frac{\hbar g}{2 \pi c k_{B}}+\frac{\hbar a}{2 \pi c k_{B}}=-\frac{\hbar G M}{2 \pi c k_{B} R^{2}}+\frac{\hbar c^{2}}{2 \pi c k_{B} \cdot 3} \Lambda R
$$


or taking a positive temperature $T_{G}$, corresponding to the gravitational acceleration $g$, by analogy with the temperature of the black holes, we obtain a negative temperature value of the vacuum $T_{v}$, which corresponds to the antigravity acceleration: $\Delta k_{B} T=k_{B} T_{G}-k_{B} T_{v}$.

Thus, the entropy $S_{v}$ is negative, which means that the effects of the vacuum can be attributed to the negative entropy, or information, that is the ability to the information arranging ordering. This explains the shift to the orderly movement of galaxies at $R>R_{Z G}$.

However, on the scale of the Universe there is a similar law accelerating recession superclusters of galaxies under the influence of dark energy (Chernin, 2013). This means that in a similar manner gravitational entropy decreases at the level of interaction of superclusters of galaxies also. Hence, the dominance of dark energy, or vacuum energy, on cosmological scales leads to the orderly movement of galaxies at the level of clusters and superclusters, reducing gravitational entropy of the Universe as a whole, as was shown earlier by the author (Bukalov, 2014). Therefore, in the Universe, which is expanding with acceleration under the influence of anti-gravity vacuum, gravitational entropy due to the contribution of gravitating matter should decrease with time. Thus, in the present Universe inside the Hubble radius are about $10^{9}$ clusters of galaxies, like the Local Group. With continued accelerated expansion of the Universe it will be only a local group. All the other galaxies will be an observer outside the event horizon, which tends to $H^{-1}=r_{\Lambda}$, the radius of de Sitter space. Thus gravitational entropy associated with the supercluster of galaxies decrease in $10^{9}$ times.

Turning to the cosmological scale and the Friedmann equation:

$$
\ddot{a}_{U}=-\frac{4 \pi}{3} G\left(\rho_{M}+3 p_{M}\right) a+\left(\rho_{V}+3 p_{V}\right) a,
$$

where $a$ is the scale factor, from $p_{M}=0, \rho_{V}=-p_{V}$ we get (Bukalov, 2014).

$$
\ddot{a}_{U}=-\frac{4 \pi G}{3}\left(\rho_{M}-2 p_{V}\right) a .
$$

When $\quad a \approx R_{H} \approx 1.36 \cdot 10^{26} \mathrm{~m}, \quad \Omega_{M} \approx 0.318, \quad \Omega_{V}=0.682$,

$$
\begin{aligned}
& \Omega_{r} \approx 5 \cdot 10^{-4}, z=0 \\
& \ddot{a}_{U}=-\frac{4 \pi}{3} G_{N} \rho_{c}\left(0.318(1+z)^{3}+\Omega_{r}(1+z)^{4}-\right. \\
& -2 \cdot 0.682) \cdot R_{H}=\frac{4 \pi}{3} G_{N} \rho_{c} \cdot 1.046 a= \\
& =3.46 \cdot 10^{-10} \mathrm{~m} \cdot \mathrm{s}^{-2} \text {. } \\
& \Delta \ddot{a}_{V}=g-2 \ddot{a}_{V} \\
& k_{B} \Delta T=k_{B} T_{g}-2 k_{B} T_{V}=-1.046 k_{B} T_{H}=-k_{B^{\prime}} T_{U} \\
& T_{U}=\frac{\hbar \ddot{a}_{U}}{2 \pi c k_{B}}=1.4 \cdot 10^{-30} \mathrm{~K} .
\end{aligned}
$$

This is the temperature of the quantum radiation of accelerated body to the outside distant observer. According to M.B. Mensky (1978) such temperature is negative. Therefore for the Universe the entropy change will be:

$$
\frac{\partial S_{U}}{\partial t} \approx \frac{S_{U}}{t_{U}} \approx-2.4 \cdot 10^{52} \mathrm{~J} / \mathrm{s}
$$

Thus, when $z<0.745, \partial S_{U} / \partial t<0$.

From our results it follows that the vacuum is a reservoir of negative entropy, or ordering, which manifests itself in the period of its domination. Therefore, the vacuum can induce an increase in ordering of the movement patterns the universe. This impact is the macroscopic quantum cosmological effect.

The existence of the Hubble flow of receding galaxies under the domination of dark energy density, vacuum energy, leads to a decrease of gravitational entropy of galaxy clusters, reducing gravitational entropy of the universe as a whole.

With the gradual disappearance of black holes with their entropy and radiation, as well as the galaxies themselves over the cosmic event horizon, the entropy of the observable universe will only decrease, and negative entropy, or the degree of ordering of information, will increase asymptotically approaching a constant value.

\section{References}

Birrell N.D., Davies P.C.W. Quantum fields in curved space. Cambridge University Press, 1982.

Bisnovatyi-Kogan G.S., Chernin A.D.: 2012, Astrophys. Space Sci., 338, 337.

Bukalov A.V.: 2014, in Proc. of $14^{\text {th }}$ International Gamow Summer School.

Chernin A., Teerikorpi P., Baryshev Yu.: 2003, Adv. Space Res., 31, 459; astro-ph/0012021.

Chernin A.D. et al.: 2007, Astron. Astrophys., 467, 933.

Chernin A.D. et al.: 2007, Astron. Astrophys. Trans., 26, 275.

Chernin A.D. et al.: 2009, Astron. Astrophys., 507, 1271.

Chernin A.D. et al.: 2012, Astron. Astrophys., 539, 4.

Chernin A.D. et al.: 2012, Astron. Rep., 56, 653.

Chernin A.D. et al.: 2013, Astron. Astrophys., 553, 101.

Chernin A.D.: 2013, UFN (Uspehi fiz. nauk), 183, 741.

Egan C.A., Lineweaver C.H.: 2010, Astrophys. J., 710, 1825; arXiv:0909.3983 [astro-ph.CO].

Brilenkov R., Eingorn M., Zhuk A.: 2015, arXiv: 1507.07234.

Eingorn M., Zhuk A.: 2012, arXiv:1205.2384.

Gibbons G.W., Hawking S.W.: 1977, Phys. Rev. D, 15, 2738.

Karachentsev I.D., Kashlbadze O.G.: 2006, Astrophys., 49, 3.

Karachentsev I.D.: 2005, Astron. J., 129, 178.

Karachentsev I.D. et al.: 2009, Mon. Not. R. Astron. Soc., 393, 1265 .

Karachentsev I.D., Karachentseva V.E., Huchtmeier W.K.: 2007, Astron. Lett., 33, 512.

Mensky M.B.: 1978, TMF, 115 (2), 215.

Perlmutter S. et al.: 1999, Astrophys. J., 517, 565.

Riess A.G. et al.: 1998, Astron. J., 116, 1009.

Teerikorpi P., Chernin A.D.: 2010, Astron. Astrophys., 516, A93. 